\title{
Towards the quantification of perioperative cardiovascular risk in the African context: A sub-analysis of the South African Surgical Outcomes Study and the African Surgical Outcomes Study
}

\author{
C S Alphonsus, ${ }^{1}$ MB ChB, FCA (SA), MMed (Anaes); H-L Kluyts, ${ }^{2}$ DMed (Anaes); V Gobin, ${ }^{3}$ MD; A Elkhogia ${ }^{4}$ FRCA; \\ F D Madzimbamuto, ${ }^{5}$ FCA (ECSA); J Tumukunde, ${ }^{6}$ MMed (Anaes); A O Omigbodun, ${ }^{7}$ MD, FRCOG; C Youssouf, ${ }^{8}$ MD; R Mehyaoui, ${ }^{9}$ MD; \\ D M Munlemvo, ${ }^{10} \mathrm{MD}$, MS; A Basenero, ${ }^{11} \mathrm{MPH}$; A Antwi-Kusi, ${ }^{12} \mathrm{MB} \mathrm{ChB}$, FWACS; D Z Ashebir, ${ }^{13} \mathrm{MD}$; A K Ndonga,${ }^{14} \mathrm{MB} \mathrm{ChB}$, \\ MMed (Surg), FCS (ECSA); Z W Ngumi, ${ }^{15}$ MB ChB, DA, FFARCS; C M Sani ${ }^{16}$ MD; A L Samateh, ${ }^{17}$ FWACS; T E Madiba, ${ }^{18} \mathrm{MB}$ ChB, \\ MMed (Surg), LLM (Medical Law), PhD, FCS (SA), FASCRS; R M Pearse, ${ }^{19}$ MD, FRCA, FFICM; B M Biccard ${ }^{20}$ MB ChB, FCA (SA), \\ MMedSci, PhD; on behalf of the African Surgical Outcomes Study (ASOS) investigators (http://samj.org.za/public/sup/15848.pdf)
}

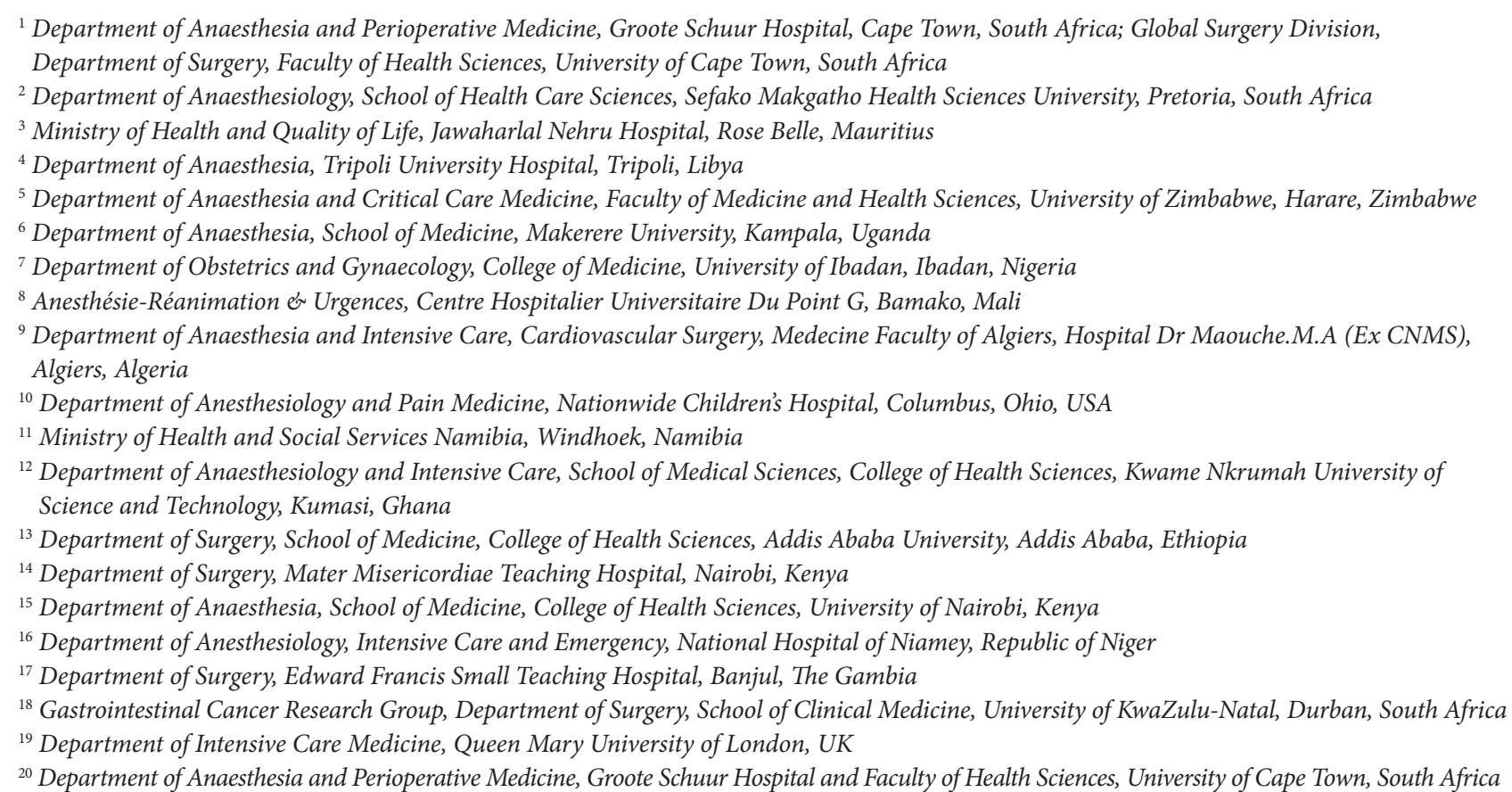

Corresponding author: C S Alphonsus (csalphonsus@gmail.com)

\begin{abstract}
Background. The burden of cardiovascular disease in patients requiring non-cardiac surgery in Africa is not known. These patients are at increased risk for postoperative cardiovascular complications.

Objectives. In this sub-study, to use data on comorbidities and surgical outcomes from two large observational studies, the South African Surgical Outcomes Study (SASOS) and the African Surgical Outcomes Study (ASOS), to investigate the prevalence of cardiovascular disease in elective surgical patients and the risk of postoperative cardiovascular complications in this population.

Methods. SASOS and ASOS were both prospective, observational cohort studies that collected data over 1 week in each participating centre. The primary outcome was in-hospital postoperative complications, which included prespecified and defined cardiovascular complications. We defined the cardiovascular disease burden of patients aged $\geq 45$ years presenting for surgery (main objective), determined the relative risk of developing postoperative cardiovascular complications (secondary objective) and assessed the utility of the Revised Cardiac Risk Index (RCRI) for preoperative cardiovascular risk stratification of elective, non-cardiac surgical patients in Africa (third objective).

Results. The primary outcome analysis of 3045 patients showed that patients with major cardiac complications were significantly older, with a higher prevalence of hypertension, coronary artery disease or congestive cardiac failure, and had undergone major surgery. In-hospital mortality for the cohort was $1.2 \%$.

Conclusions. The substantial burden of cardiovascular disease in patients presenting for non-cardiac surgery in Africa is shown in the principal findings of this study. The RCRI has moderate discrimination for major cardiac complications and major adverse cardiac events in African patients undergoing non-cardiac surgery.
\end{abstract}

S Afr Med J 2021;111(11):1065-1069. https://doi.org/10.7196/SAMJ.2021.v111i11.15848 
Cardiovascular disease is the leading cause of death worldwide. In surgical patients, the Vascular Events in Noncardiac Surgery Patients Cohort Evaluation (VISION) study ${ }^{[1]}$ showed that patients with cardiovascular comorbidities had an increased incidence of perioperative cardiac complications. The 30-day mortality rate was $2 \%$ in patients aged $\geq 45$ years, ${ }^{[1]}$ and $8 \%$ had significant myocardial injury that contributed to subsequent morbidity. ${ }^{[2]}$

The number of patients presenting for surgery in Africa with cardiovascular disease is not known. Two large observational studies, the South African Surgical Outcomes Study (SASOS) ${ }^{[3]}$ and the African Surgical Outcomes Study (ASOS), ${ }^{[4]}$ collected demographic and clinical data that could provide insight into this problem. Both studies were conducted in a similar manner, as a 7-day snapshot of patients aged $\geq 18$ years undergoing any inpatient surgery. The South African (SA) cohort comprised 3927 patients, and the African cohort 11422 patients from 25 countries. The data presented in this article will show the burden of cardiovascular disease in patients presenting for elective surgery and the risk of developing postoperative cardiovascular complications.

Defining perioperative risk requires steps to identify high-risk patients who will develop adverse outcomes. This is done prior to surgery through risk stratification, where patient comorbidities and clinical data are used in risk scores and indices. The Revised Cardiac Risk Index (RCRI) is a commonly used risk stratification tool and is recommended in international guidelines for preoperative cardiovascular risk stratification. ${ }^{[5-7]}$ However, the RCRI only provides moderate discrimination to predict postoperative cardiac complications. ${ }^{[8,9]}$ The RCRI was derived from a single-centre cohort study, ${ }^{[10]}$ and a systematic review of the utility of the RCRI including 24 cohort studies from across the globe did not include studies from African countries. ${ }^{[8]}$ The utility and calibration of the RCRI are therefore unknown in African surgical patients.

\section{Objectives}

In this sub-study, we defined the cardiovascular disease burden in patients presenting for surgery on the African continent and the incidence of postoperative cardiovascular complications. We also used the RCRI to quantify perioperative risk for complications in these patients.

\section{Methods}

Data were pooled from two large observational studies. SASOS was registered on ClinicalTrials.gov (NCT02141867) and collected data from 19 May to 26 May 2014 in 50 hospitals in the public sector. ASOS was registered on the South African National Health Research Database (KZ_2015RP7_22) and ClinicalTrials.gov (NCT03044899). All participating sites received local ethics and regulatory approval for participating in SASOS and ASOS. ASOS used a convenience sampling strategy in participating African countries between February and May 2016. Each country collected data from at least 10 hospitals, or at least half the surgical centres if there were $<10$ surgical hospitals in the country. Data on all patients undergoing elective and emergency surgery procedures that required at least an overnight stay in hospital were collected. ${ }^{[4]}$ The primary objective in SASOS was in-hospital mortality and the need for critical care admission in patients, although some hospitals collected in-hospital complications for the International Surgical Outcomes Study (ISOS). ${ }^{[1]}$ The primary objective in ASOS was in-hospital complications and the secondary objective was in-hospital mortality. Ethics approval for this sub-study was given by the Human Ethics Research Committee, University of Cape Town, on 6 September 2019 (ref. no. HREC 615/2019).
In this sub-study, the primary objective was to define the cardiovascular disease burden of patients aged $\geq 45$ years presenting for surgery in the combined SASOS and ASOS data set. Data were pooled from hospitals which collected data on the following comorbidities associated with adverse cardiovascular outcomes: hypertension, diabetes mellitus, stroke, coronary artery disease, congestive cardiac failure and chronic renal failure. ${ }^{[6,10]}$

The secondary objective was to determine the relative risk (RR) of developing postoperative cardiovascular outcomes in the SASOS/ ASOS cohort. The cardiovascular outcomes defined in SASOS/ ASOS were myocardial infarction, arrhythmias, pulmonary oedema, pulmonary embolism, stroke and cardiac arrest. As a comparison, we also used the outcomes defined in the RCRI, which are myocardial infarction, pulmonary oedema, ventricular fibrillation or primary cardiac arrest, and complete heart block. The data collected in SASOS/ASOS were not standardised for cardiovascular outcomes, so the definitions are not uniform but overlap with the RCRI in some instances.

The third objective was to determine the utility of the RCRI for preoperative cardiovascular risk stratification of elective, non-cardiac surgical patients in Africa aged $\geq 45$ years. The preoperative RCRI risk factors were defined in the original derivation cohort by Lee et al. ${ }^{[10]}$ as a history of coronary artery disease, a history of congestive cardiac failure, preoperative use of insulin, a history of stroke, a preoperative creatinine level $>2 \mathrm{mg} / \mathrm{dL}$, and high-risk surgery. The equivalent definitions of these risk factors in ASOS were a history of coronary artery disease, a history of congestive cardiac failure, a history of diabetes mellitus, a history of chronic renal disease, and major surgery. The outcome for the third objective was a composite of myocardial infarction, pulmonary oedema, and cardiac arrest and arrhythmia.

The discrimination of the RCRI for cardiac complications was described using the receiver operating characteristic (ROC) and area under the curve (AUC). The calibration between the original derivation cohort ${ }^{[10]}$ and the ASOS cohort ${ }^{[4]}$ was compared using the RR for cardiac complications in the African cohort for each RCRI risk factor. The likelihood ratios for low- (0 RCRI risk factors), intermediate- (1 - 2 RCRI risk factors) and high-risk ( $\geq 3$ RCRI risk factors) categories were calculated.

Data are presented as number (proportion). In order to compare the characteristics of the patient groups with and without cardiac complications, the categorical variables were compared using the $\chi^{2}$ or Fisher's exact test, as appropriate. Continuous variables were tested for normality and summarised using mean and standard deviation or median and interquartile range, as appropriate. An independentsamples $t$-test was used to compare normally distributed data. Risk is presented as RR with $95 \%$ confidence intervals (CIs) using MedCalc (https://www.medcalc.org/calc/relative_risk.php). Statistical analyses were performed using the Statistical Package for the Social Sciences (SPSS) version 25 (IBM, USA). The level of significance ( $\alpha$ ) was set at 0.05 .

\section{Results}

The primary outcome analysis included 3045 patients (Fig. 1), with 810 patients having been excluded owing to missing data. In this cohort, 2048 patients were from South Africa: 989 patients from SASOS and 1059 from ASOS. Table 1 shows the baseline characteristics of the cohort. Patients with major cardiac complications were significantly older, with a higher prevalence of hypertension, coronary artery disease or congestive cardiac failure, and underwent major surgery. In-hospital mortality for the cohort was $1.2 \%$. 
The prevalence of cardiovascular disease in the population was as follows: hypertension $41.3 \%$, coronary artery disease $4.7 \%$, congestive cardiac failure $1.9 \%$, diabetes mellitus $15.0 \%$, stroke $1.4 \%$ and chronic renal disease $2.8 \%$ (Table 1 ). Table 2 shows the relative risk of developing cardiovascular complications using two different definitions. Coronary artery disease, congestive cardiac failure and major surgery were significantly associated with adverse cardiovascular events under both the SASOS/ASOS and RCRI outcome definitions. Stroke, diabetes and chronic renal disease were not associated with cardiovascular outcomes.

The incidence of postoperative cardiovascular complications according to the RCRI definition is shown in Tables 3 and 4 . Missing data necessary to generate the RCRI outcome resulted in 28 patients with the data necessary for these analyses. The incidence of postoperative cardiovascular complications per point increase in the RCRI was $0.7 \%, 1.5 \%, 4.3 \%$ and $15 \%$ for $0,1,2$ and $\geq 3$ points, respectively (Table 3). The original index in 1999 showed a risk estimate of $0.4 \%, 0.9 \%, 6.6 \%$ and $11 \% .{ }^{[10]}$

The incidence of postoperative cardiovascular complications according to the RCRI categories was $0.73 \%$ in the low-risk category, $1.97 \%$ in the intermediate-risk category, and $15 \%$ in the high-risk category (Table 4). The likelihood ratios for each risk category are shown.

The discrimination of the RCRI is shown in the ROC curve for major cardiac complications (Fig. 2). It shows an AUC of 0.68 (95\% CI $0.57-0.79)$. In the original cohort, the AUC was $0.78(95 \%$ CI $0.73-0.82) \cdot{ }^{[10]}$

\section{Discussion}

This study examined the cardiovascular disease burden of patients presenting for non-cardiac surgery on the African continent. In this cohort, a large proportion of patients had hypertension (41.3\%), a history of smoking (18.9\%) and diabetes mellitus (15.0\%). Patients who were older with hypertension, coronary artery disease or congestive cardiac failure or were exposed to major surgery had an increased risk of postoperative cardiac complications. The RCRI demonstrated moderate discrimination for postoperative cardiovascular complications in African patients undergoing noncardiac surgery, with an AUC of 0.68 .

Previous studies have identified the concerning increase in noncommunicable disease on the African continent. ${ }^{[12]}$ Hypertension, smoking and diabetes mellitus are known risk factors for

Table 1. African surgical patients aged $\geq 45$ years

\begin{tabular}{|c|c|c|c|c|}
\hline Risk factor & Total patients & $\begin{array}{l}\text { Patients with major } \\
\text { cardiac complications* }\end{array}$ & $\begin{array}{l}\text { Patients without major } \\
\text { cardiac complications }\end{array}$ & $p$-value \\
\hline Age (years), mean (SD) & $60.83(10.88)$ & $67.39(12.24)$ & $60.62(10.77)$ & $<0.001$ \\
\hline Female, $n / N(\%)$ & $1172 / 2235(52.4)$ & $22 / 38(57.9)$ & $1150 / 2197(52.3)$ & 0.52 \\
\hline Smoking, $n / N(\%)$ & $417 / 2202(18.9)$ & $4 / 37(10.8)$ & $413 / 2165(19.1)$ & 0.29 \\
\hline \multicolumn{5}{|l|}{ Comorbidities, $n / N(\%)$} \\
\hline Hypertension & $822 / 1988(41.3)$ & $21 / 35(60.0)$ & $801 / 1953(41.0)$ & 0.04 \\
\hline Coronary artery disease & $106 / 2232(4.7)$ & $7 / 38(18.4)$ & $99 / 2194(4.5)$ & 0.002 \\
\hline Congestive cardiac failure & $43 / 2232(1.9)$ & $7 / 38(18.4)$ & $36 / 2194(1.6)$ & $<0.0001$ \\
\hline Stroke & $32 / 2232(1.4)$ & $1 / 38(2.6)$ & $31 / 2194(1.4)$ & 0.43 \\
\hline Diabetes mellitus & $335 / 2232(15.0)$ & $10 / 38(26.3)$ & $325 / 2194(14.8)$ & 0.63 \\
\hline Chronic renal disease & $56 / 1990(2.8)$ & $1 / 35(2.9)$ & $55 / 1995(2.8)$ & 1.00 \\
\hline Major surgery, $n / N(\%)$ & $637 / 2235(28.5)$ & $23 / 38(60.5)$ & $614 / 2197(27.5)$ & $<0.0001$ \\
\hline \multicolumn{5}{|l|}{ ASA, $n / N(\%)$} \\
\hline Class 1 & $672 / 2220(30.2)$ & $2 / 37(5.4)$ & $670 / 2183(30.7)$ & 0.0004 \\
\hline Class 2 & $1117 / 2220(50.3)$ & $12 / 37(32.4)$ & $1105 / 2183(50.6)$ & 0.03 \\
\hline Class 3 & $403 / 2220(18.2)$ & $19 / 37(51.4)$ & $384 / 2183(17.6)$ & 0.0001 \\
\hline Class 4 & $28 / 2220(1.3)$ & $4 / 37(10.8)$ & $24 / 2183(1.1)$ & 0.001 \\
\hline \multicolumn{5}{|l|}{ Type of procedure, $n / N(\%)$} \\
\hline Orthopaedic & $420 / 2234(18.8)$ & $7 / 38(18.4)$ & $413 / 2196(18.8)$ & 1.0 \\
\hline Breast & $100 / 2234(4.5)$ & $2 / 38(5.3)$ & $98 / 2196(4.5)$ & 0.69 \\
\hline Obstetrics $^{\dagger}$ & $4 / 2234(0.2)$ & 0 & $4 / 2196(0.2)$ & 0.64 \\
\hline Gynaecology & $272 / 2234(12.2)$ & $3 / 38(7.9)$ & $269 / 2196(12.2)$ & 0.62 \\
\hline Upper gastrointestinal & $82 / 2234(3.7)$ & $3 / 38(7.9)$ & $79 / 2196(3.6)$ & 0.16 \\
\hline Lower gastrointestinal & $175 / 2234(7.8)$ & $2 / 38(5.3)$ & $173 / 2196(7.9)$ & 0.76 \\
\hline Hepatobiliary & $89 / 2234(4.0)$ & $1 / 38(2.6)$ & $88 / 2196(4.0)$ & 1.0 \\
\hline Urology & $345 / 2234(15.4)$ & $5 / 38(13.2)$ & $340 / 2196(15.5)$ & 0.82 \\
\hline Vascular & $54 / 2234(2.4)$ & $3 / 38(7.9)$ & $51 / 2196(2.3)$ & 0.06 \\
\hline Head and neck & $151 / 2234(6.8)$ & $3 / 38(7.9)$ & $148 / 2196(6.7)$ & 0.74 \\
\hline Plastics & $127 / 2234(5.7)$ & $1 / 38(2.6)$ & $126 / 2196(5.7)$ & 0.72 \\
\hline Thoracic & $2 / 2234(1.6)$ & $2 / 38(5.3)$ & $33 / 2196(1.5)$ & 0.12 \\
\hline Neurosurgery & $42 / 2234(1.9)$ & $2 / 38(5.3)$ & $40 / 2196(1.8)$ & 0.16 \\
\hline Other & $306 / 2234(13.2)$ & 0 & $306 / 2196(13.9)$ & 0.0007 \\
\hline
\end{tabular}




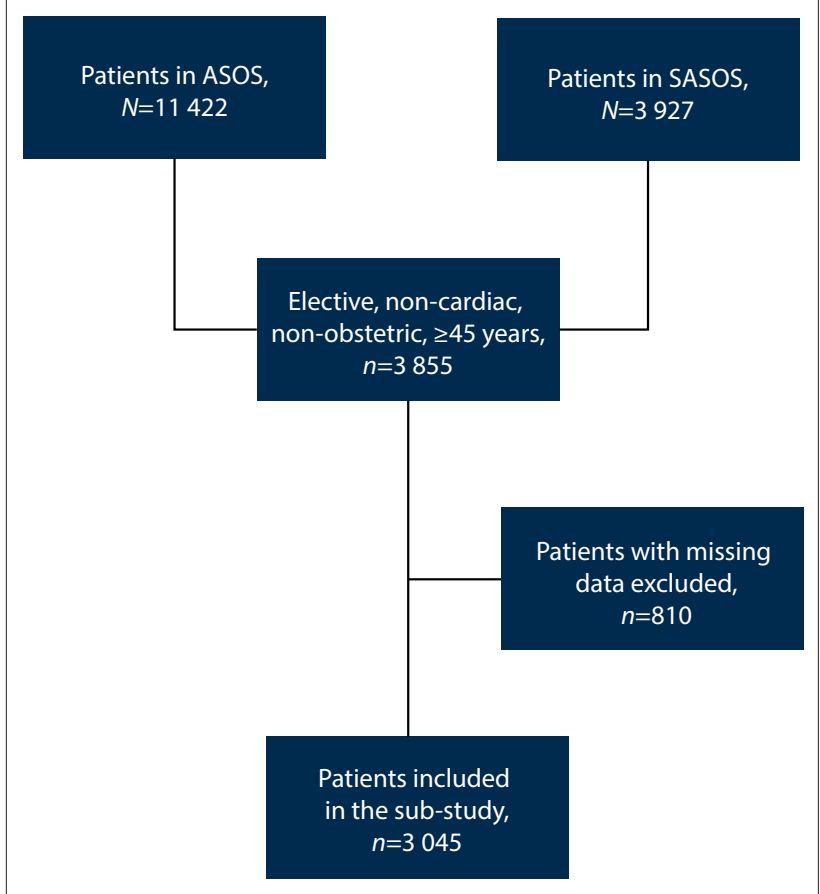

Fig. 1. STROBE diagram of the ASOS and SASOS patients included in the sub-study. $($ ASOS = African Surgical Outcomes Study; SASOS $=$ South African Surgical Outcomes Study.) atherothrombotic cardiovascular disease, and this cohort had a very high prevalence of these risk factors. ${ }^{[12]}$ Additionally, in previous studies, the overall management of these cardiovascular risk factors from diagnosis to treatment and long-term control was shown to be poor. ${ }^{[12]}$ This poor management is probably due to insufficient human and financial resources and lack of infrastructure in low- and middleincome countries. ${ }^{[13]} \mathrm{A}$ recent review of cardiovascular disease in subSaharan Africa notes a paucity of data on the prevalence of ischaemic heart disease and cardiac failure. ${ }^{[12]}$ The data on mortality caused by ischaemic heart disease and cardiac failure in sub-Saharan Africa are thought to be an underestimate owing to lack of biomarker and other diagnostic capabilities. ${ }^{[1,15]}$ In the present sub-study, cardiac failure and ischaemic heart disease were significant causes of postoperative cardiovascular complications. Stroke and diabetes mellitus did not show a significant association with complications (Table 2); however, it is likely that this is due to an inadequate sample size in this substudy to demonstrate this association.

A large proportion of the data on cardiovascular disease in in the present study came from the SA cohort of patients. Despite this limitation, the main message is that cardiovascular comorbidities present an additional risk for postoperative cardiovascular complications. This increased risk was shown across different populations in the VISION study. ${ }^{[1]}$

In order to relate our findings to the commonly used tool for cardiovascular risk stratification, the RCRI, we applied the RCRI in this cohort of patients. However, we could not perform an external validation of the RCRI in the SASOS/ASOS cohort owing

Table 2. Relative risk for cardiovascular complications using SASOS/ASOS outcome definition and RCRI outcome definition

\begin{tabular}{l}
\hline Comorbidities \\
\hline Female \\
Smoking \\
Hypertension \\
Coronary artery disease \\
Congestive cardiac failure \\
Stroke \\
Diabetes mellitus \\
Chronic renal disease \\
Major surgery
\end{tabular}

SASOS/ASOS definition
1.10 (95\% CI $0.55-2.22) ; p=0.77$
$0.52(95 \%$ CI $0.18-1.46) ; p=0.21$
$2.13(95 \%$ CI $1.09-4.16) ; p=0.03$
4.5 (95\% CI $2.04-10.04) ; p=0.0002$
11.5 (95\% CI $5.36-24.64) ; p<0.0001$
1.86 (95\% CI $0.26-13.13) ; p=0.53$
$2.02(95 \%$ CI $0.99-4.12) ; p=0.053$
$1.02(95 \%$ CI $0.14-7.29) ; p=0.99$
3.85 (95\% CI $2.02-7.32) ; p<0.0001$

RCRI definition

1.10 (95\% CI $0.55-2.22) ; p=0.77$

0.66 ( $95 \%$ CI $0.23-1.88) ; p=0.43$

1.89 ( $95 \%$ CI $0.9-3.97) ; p=0.09$

4.8 (95\% CI $2.02-11.54) ; p=0.0004$

12.27 (95\% CI $5.31-28.38) ; p<0.0001$

1.06 ( $95 \%$ CI $0.07-17.01) ; p=0.97$

1.36 ( $95 \%$ CI 0.56 - 3.28); $p=0.50$

1.29 (95\% CI $0.18-9.3) ; p=0.80$

$3.44(95 \%$ CI $1.7-6.98) ; p=0.0006$

SASOS = South African Surgical Outcomes Study; ASOS = African Surgical Outcomes Study; RCRI = Revised Cardiac Risk Index; CI = confidence interval.

Table 3. Risk estimate for each point of the RCRI ( $\geq 45$ years) for composite outcome in the SASOS/ASOS cohort compared with the original RCRI cohort*

\begin{tabular}{lll}
\hline RCRI & African surgical cohort risk estimate, $\%$ & Original 1999 RCRI cohort, \% \\
\hline 0 point & $0.7(95 \%$ CI $0.23-1.20)$ & $0.4(95 \%$ CI $0.05-1.50)$ \\
1 point & $1.5(95 \%$ CI $0.62-2.4)$ & $0.9(95 \%$ CI $0.30-2.10)$ \\
2 points & $4.3(95 \%$ CI $0.90-7.60)$ & $6.6(95 \%$ CI $3.90-10.30)$ \\
$\geq 3$ points & $15(95 \%$ CI $0.65-30.70)$ & $11(95 \%$ CI $5.80-18.40)$ \\
& & \\
SASOS $=$ South African Surgical Outcomes Study; ASOS = African Surgical Outcomes Study; RCRI = Revised Cardiac Risk Index; CI = confidence interval. \\
${ }^{*}$ Composite outcome of myocardial infarction, pulmonary oedema and cardiac arrest and arrhythmia.
\end{tabular}

Table 4. Risk estimate per category of the RCRI

\begin{tabular}{llll}
\hline RCRI category & $n / N$ & Risk estimate, $\%$ & LR \\
\hline Low risk (0 points) & $8 / 1092$ & $0.73(95 \%$ CI $0.23-1.24)$ & 0.52 \\
Intermediate risk ( 1 - 2 points) & $17 / 863$ & $1.97(95 \%$ CI $1.04-2.89)$ & 1.4 \\
High risk ( $\geq 3$ points) & $3 / 20$ & $15(95 \%$ CI $0-30.65)$ & 10.6
\end{tabular}

RCRI $=$ Revised Cardiac Risk Index; $n=$ number affected; $N=$ total population; $\mathrm{CI}=$ confidence interval; $\mathrm{LR}=$ likelihood ratio 


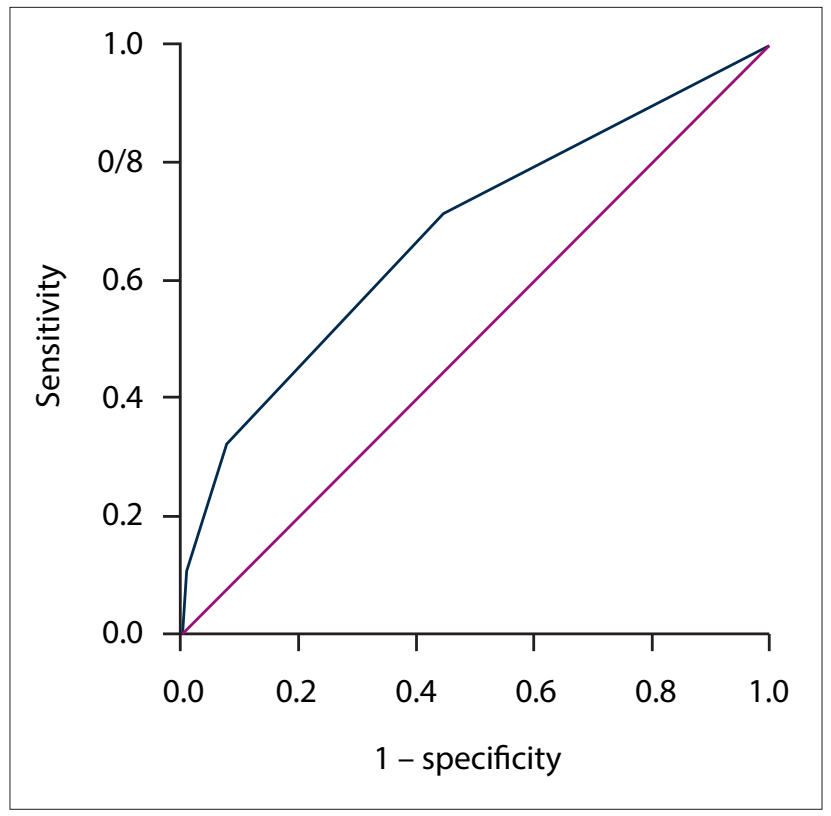

Fig. 2. Receiver operating characteristic curve of the Revised Cardiac Risk Index and major cardiac complications in elective African surgical patients aged $\geq 45$ years.

to differences in risk factor and outcome definitions. As the SASOS/ ASOS studies did not use routine troponin screening as part of postoperative detection of myocardial injury after non-cardiac surgery, it is likely that the incidence of postoperative cardiovascular complications is under-reported in our cohort. ${ }^{[2,16]}$ Nevertheless, the presence of coronary artery disease, congestive cardiac failure and major surgery were strong predictors of postoperative cardiovascular complications despite different study outcome definitions.

The utility of the RCRI to risk-stratify patients at cardiovascular risk is becoming more controversial. It appears to provide good discrimination for patients at low risk (with no RCRI risk factors), as reflected by a low likelihood ratio. ${ }^{[9]}$ However, in the most concerning group, which is patients with one or more RCRI risk factors who are consequently at higher risk for complications, the performance of the RCRI is poor. ${ }^{[9]}$ As a result, the Canadian Cardiovascular Society ${ }^{[7]}$ and the American College of Cardiology/American Heart Association $^{[5]}$ recommend further risk stratification of patients with one or more risk factors. The data from the SASOS/ASOS cohort would also suggest that the utility of the RCRI in an African cohort is at best moderate, and unlikely to be satisfactory for the risk stratification of patients with one or more risk factors. Further recommendation is limited in this sub-study by the small sample size and wide CIs in the high-risk category of patients.

Future studies in this cohort of patients with cardiovascular comorbidities need to focus on cardiovascular outcomes, risk stratification and optimisation before surgery.

\section{Conclusions}

In this sub-study, we have shown that a substantial proportion of patients with cardiovascular disease present for non-cardiac surgery on the African continent. These comorbidities contribute to postoperative complications and adverse outcomes. The RCRI potentially provides moderate discrimination for preoperative cardiac risk stratification in African patients undergoing non-cardiac surgery.

Declaration. The research for this study was done in partial fulfilment of the requirements for CSA's PhD (Anaesthesia) at the University of Cape Town. Acknowledgements. CSA thanks the Global Surgery Division, University of Cape Town, for support through the Global Surgery Fellowship grant as part of the South African Medical Research Council Midcareer Scientist Award. Author contributions. CSA: protocol development, data collection, data collation, data analysis, wrote manuscript. H-LK, VG, AE, FDM, JT, AOO, CY, RM, DMM, AB, AA-K, DZA, AKN, ZWN, CMS, ALS, TEM, RMP, BMB: protocol development, data collection, data collation, reviewed drafts of manuscript.

Funding. Funding was received from the South African Medical Research Council. This study was an investigator initiated and led study, and the funding body therefore had no role in the design, running, analysis, writing, or publication of the results.

Conflicts of interest. RMP has received research grants and/or honoraria from Edwards Lifesciences, Intersurgical and GlaxoSmithKline. The other authors declare that they have no conflict of interest.

1. Devereaux PJ, Chan MT, Alonso-Coello P, et al. Association between postoperative troponin level and 30-day mortality among patients undergoing noncardiac surgery. JAMA 2012;307(21):2295-2304. https://doi.org/10.1001/jama.2012.5502

2. Botto F, Alonso-Coello $\mathrm{P}$, Chan MT, et al. Myocardial injury after noncardiac surgery: A large, international, prospective cohort study establishing diagnostic criteria, characteristics, predictors, an 30-day outcomes. Anesthesiology 2014;120(3):564-578. https://doi.org/10.1097/aln.0000000000000113

3. Biccard BM, Madiba TE, South African Surgical Outcomes Study I. The South African Surgical Outcomes Study: A 7-day prospective observational cohort study. S Afr Med J 2015;105(6):465-475. https://doi.org/10.7196/SAMI.9435

4. Biccard BM, Madiba TE, Kluyts HL, et al. Perioperative patient outcomes in the African Surgical Outcomes Study: A 7-day prospective observational cohort study. Lancet 2018;391(10130):1589-1598. 10.7196/SAMJ.9435

5. Fleisher LA, Fleischmann KE, Auerbach AD, et al. 2014 ACC/AHA guideline on perioperative cardiovascular evaluation and management of patients undergoing noncardiac surgery: A report of the American College of Cardiology/American Heart Association Task Force on Practice Guidelines. J Am Coll Cardiol 2014;64(22):e77-el37. https://doi.org/10.1161/cir.000000000000010

6. Kristensen SD, Knuuti J, Saraste A, et al. 2014 ESC/ESA guidelines on non-cardiac surgery: Cardiovascular assessment and management: The Joint Task Force on non-cardiac surgery: Cardiovascular assessmen and management of the European Society of Cardiology (ESC) and the European Society Anaesthesiology (ESA). Eur Heat J 2014,35(35):2383-2431. h/ps.//doi.org/10.109/eurheartj/ehu282

7. Duceppe E, Parlow J, MacDonald P, et al. Canadian Cardiovascular Society guidelines on perioperative cardiac risk assessment and management for patients who undergo noncardiac surgery. Can J Cardiol 2017;33(1):17-32. https://doi.org/10.1016/.j.cca.2016.09.008

8. Ford MK, Beattie WS, Wijeysundera DN. Systematic review: Prediction of perioperative cardiac complications and mortality by the Revised Cardiac Risk Index. Ann Intern Med 2010;152(1):26-35 https://doi.org/10.7326/0003-4819-152-1-201001050-00007

9. Biccard B. Proposed research plan for the derivation of a new Cardiac Risk Index. Anesth Analg 2015;120(3):543-553. https://doi.org/10.1213/ane.0000000000000598

10. Lee TH, Marcantonio ER, Mangione CM, et al. Derivation and prospective validation of a simple index for prediction of cardiac risk of major noncardiac surgery. Circulation 1999;100(10):1043-1049. https:// doi.org/10.1161/01.cir.100.10.1043

11. International Surgical Outcomes Study Group. Global patient outcomes after elective surgery: Prospective cohort study in 27 low-, middle- and high-income countries. Br J Anaesth 2016;117(5):601 609. https://doi.org/10.1093/bja/aew316

12. Yuyun MF, Sliwa K, Kengne AP, Mocumbi AO, Bukhman G. Cardiovascular diseases in sub-Saharan Africa compared to high-income countries: An epidemiological perspective. Glob Heart 2020;15(1):15. http://doi.org/10.5334/gh.403

13. Nyaaba GN, Stronks K, de-Graft Aikins A, Kengne AP, Agyemang C. Tracing Africa’s progress towards implementing the Non-Communicable Diseases Global Action Plan 2013 - 2020: A synthesis of WHO
country profile reports. BMC Public Health 2017;17(1):297 https://doi.org/10.1186/s12889-017-4199-6 Country profile reports. BMC Public Health 2017;17(1):297. https://doi.org/10.1186/s12889-017-4199-6
4. Nkoke C, Luchuo EB. Coronary heart disease in sub-Saharan Africa. Still rare misdiagned or Nkoke C, Luchuo EB. Coronary heart disease in sub-Saharan Africa: Still rare, misdiagnosed o
underdiagnosed? Cardiovasc Diagn Ther 2016;6(1):64-66. https://doi.org/10.3978/jissn 2223 underdiagnosed?

15. Carlson S, Duber HC, Achan J, et al. Capacity for diagnosis and treatment of heart failure in sub-Saharan Africa. Heart 2017;103(23):1874-1879. https://doi.org/10.1136/heartinl-2016-310913

16. Writing Committee for the VISION Study Investigators. Association of postoperative high-sensitivity Writing Committee for the VISION Study Investigators. Association of postoperative high-sensitivity
troponin levels with myocardial injury and 30-day mortality among patients undergoing noncardiac surgery. JAMA 2017;317(16):1642-1651. https://doi.org/10.1001/jama.2017.4360 